\title{
Desenvolvimento tecnológico de hidrogéis a partir de nanoemulsão contendo clotrimazol em associação com o óleo de melaleuca
}

\author{
Thamiris Coimbra Paines, Julia Andrighetto de Lima, Julia Weber, Fernanda Cramer \\ Flores, Cristiane de Bona da Silva
}

\begin{abstract}
Programa de Pós-Graduação em Ciências Farmacêuticas, Universidade Federal de Santa Maria, RS, Brasil
miriscp@hotmail.com; juliaadelima@gmail.com; weber.julia@gmail.com; weber.julia@gmail.com; csbona@smail.ufsm.br
\end{abstract}

\section{Resumo}

O clotrimazol é um antifúngico imidazólico de uso tópico indicado para o tratamento de micoses superficiais, principalmente aquelas causadas por leveduras do gênero Candida. O óleo essencial de Melaleuca alternifolia (óleo de melaleuca) apresenta conhecidas propriedades anti-inflamatória, antiviral, antibacteriana e antifúngica. No presente trabalho, nanoemulsões contendo clotrimazol e o óleo de melaleuca foram preparadas, a fim de obter uma forma farmacêutica semissólida de base aquosa contendo o nanocarreador proposto. As nanoemulsões foram preparadas pelo método de emulsificação espontânea e apresentaram tamanho manométrico (150 a $380 \mathrm{~nm}$ ), baixo indice de polidispersão $(<0,13)$, potencial zeta negativo e teor de fármaco próximo ao teórico $(1,02 \pm 0,92 \mathrm{mg} / \mathrm{mL})$, as quais se mantiveram estáveis após 30 dias de armazenamento a temperatura ambiente. Na avaliação da atividade antifúngica frente à leveduras de C. albicans, o fármaco manteve sua atividade. Posteriormente, as nanoemulsões foram incorporadas em géis de Carbopol ${ }^{\circledR}$ Ultrez e estes foram avaliados, os quais apresentaram características adequadas para aplicação tópica: $p H$ levemente ácido $(6,2$ a 6,5), comportamento de fluxo não-newtoniano $e$ plástico e teor de fármaco próximo a 100\%, demonstrando a viabilidade tecnológica de sua preparação. Além disso, o hidrogel contendo o fármaco nanoestruturado apresentou um controle maior de liberação do clotrimazol quando comparado ao hidrogel contendo o fármaco não associado.

Palavras-chave: Clotrimazol. Óleo de melaleuca. Nanoemulsões. Hidrogéis.

\begin{abstract}
Clotrimazole is an antifungal imidazole of topical use indicated for the treatment of superficial mycoses, particularly those caused by Candida species. The essential oil of Melaleuca alternifolia presents notorious anti-inflammatory, antiviral, antibacterial and antifungal properties. In this study, nanoemulsions containing clotrimazole and melaleuca oil were prepared in order to obtain a water-based semisolid dosage form containing the nanocarrier proposed. The nanoemulsions were prepared by spontaneous emulsification method and showed nanometric mean size $(150$ to $380 \mathrm{~nm})$, low polydispersity $(<0.13)$, negative zeta potential and drug content close to theoretical concentration $(1.02 \pm 0.92 \mathrm{mg} / \mathrm{mL})$. The formulations were stable during 30 days of storage at room temperature. In the evaluation of antifungal activity against Candida albicans, clotrimazole maintained its activity. Furthermore, nanoemulsionswere incorporated into hydrogels of Carbopol ${ }^{\circledR}$ Ultrez and these were evaluated, showing suitable characteristics for topical application: slightly acid pH (6.2 to 6.5), non-Newtonian and plastic flow behavior, and drug content close to $100 \%$, demonstrating the technological feasibility of its preparation. In addition, the hydrogel containing the nanostructure showed a greater drug release control in comparison with the hydrogel containing the drug not associate.
\end{abstract}

Keywords: Clotrimazole. Tea tree oil. Nanoemulsions. Hydrogels. 


\section{INTRODUÇÃO}

As micoses superficiais são infecções fúngicas que afetam mais de $25 \%$ da população mundial, sendo que leveduras do gênero Candida são importantes agentes causadores (HAV LICKOVA et al., 2008). O clotrimazol - 1-[(2-clorofenil) difenilmetil]-1H-imidazol (BRITISH PHARMACOPEIA I, 2010), é um antifúngico imidazólico de amplo espectro que atua pela inibição da síntese do ergosterol, componente essencial da membrana celular fúngica, por inativar a enzima lanosterol 14- $\alpha$-desmetilase (GEORGOPAPAD AKOU et al., 1998). É indicado contra dermatomicoses superficiais, infecções causadas por Candida spp. e pitiríase versicolor. Seu uso é limitado a formulações de aplicação tópica, devido a sua toxicidade e efeitos adversos, como náuseas, vômitos, dor abdominal e diarreia, quando administrado pela via oral (BURGESS \& BODEY, 1972; GUPTA et al., 1998). No entanto, pode causar ardência, urticária e erupção cutânea, tanto pela via tópica, como pela vaginal. Além disso, sua absorção local é reduzida devido a sua baixa solubilidade em água (CHANG et al., 2002; SOBEL et al., 2007; SOUTO \& MÜLLER, 2007).

O óleo essencial de Melaleuca alternifolia (óleo de melaleuca e, em inglês, tea tree oil) é popularmente conhecido como um produto de uso tópico devido suas propriedades antifúngica, antiviral, antibacteriana, antiprotozoária e anti-inflamatória (CARSON et al., 2006). Caracteriza-se por uma mistura complexa de cerca de 100 diferentes compostos, principalmente, de monoterpenos e seus álcoois correspondentes. Seu principal componente, o terpinen-4-ol, seguido de $\alpha$ e $\gamma$-terpineno, caracterizam-se como promotores de penetração cutânea (REICHLING et al., 2006). No entanto, as características físico-químicas do óleo apresentam certas adversidades que comprometem o seu uso, e fatores como luz, calor, exposição ao ar e umidade podem afetar a sua estabilidade. Além disso, seu caráter lipofílico conduz a problemas de miscibilidade em produtos de base de água (CARSON et al., 2006).

A nanotecnologia abrange estruturas de diâmetro inferior a $1 \mu \mathrm{m}$. A utilização terapêutica de sistemas nanoestruturados tem sido amplamente pesquisada, visto que o tamanho reduzido dos mesmos confere diversas vantagens às formulações farmacêuticas. Dentre essas vantagens, se encontram a melhora na farmacocinética e na biodistribuição de agentes terapêuticos, na solubilidade de substâncias lipofílicas (MISHRA et al., 2010), além de possibilitar uma liberação controlada e segura do fármaco por via cutânea (GUTERRES et al., 2007), proteger as substâncias contra agentes externos, como a proteção do óleo de melaleuca frente à volatilização (FLORES et al., 2011) e melhorar a fotoestabilidade de fármacos como o clotrimazol (SANTOS et al., 2013).

Entre os nanocarreadores, as nanoemulsões são gotículas de óleo em água, estabilizadas por tensoativos, que apresentam, geralmente, um tamanho nanométrico de até 500 nm (ANTON et al., 2008). Estes carreadores mostram-se eficientes sistemas de liberação cutânea, garantindo uma distribuição uniforme da formulação na pele devido ao tamanho reduzido de suas gotículas (SONNEVILLE-AUBRUN et al., 2004). Mirza e colaboradores (2013) avaliaram a atividade antifúngica de géis contendo nanoemulsões de itraconazol e óleo de melaleuca frente à candidíase vulvovaginal, propondo uma nova ferramenta para o tratamento das afecções vaginais.

Considerando o exposto, o presente trabalho consiste no desenvolvimento de uma formulação semissólida do tipo hidrogel contendo clotrimazol e o óleo de melaleuca associados à nanoemulsão. Esta estratégia tecnológica se baseia na possibilidade de obtenção de uma preparação semissólida de base aquosa contendo um óleo essencial com importantes propriedades farmacológicas, em associação com o fármaco em estudo, a fim de contornar obstáculos da terapia atual, como incidência de efeitos indesejáveis e permanência das substâncias ativas no local de ação A formulação foi avaliada em relação as suas propriedades físico-químicas e liberação in vitro.

\section{MATERIAL E MÉTODOS}

\subsection{Material}

O clotrimazol (CTZ - 100,39\%) e Carbopol ${ }^{\circledR}$ Ultrez foram obtidos da Deg (São Paulo, Brasil). O óleo essencial de melaleuca foi obtido de Lazlo Aromaterapia (Belo Horizonte, Brasil). Monooleato de sorbitano (Span $\left.{ }^{\circledR} 80\right)$ foi adquirido da Sigma (São Paulo, Brasil); polissorbato 80 (Tween ${ }^{\circledR} 80$ ) foi fornecido pela Delaware (Porto Alegre, Brasil); metanol grau cromatográfico foi adquirido da Tedia (São Paulo, Brasil). Todos os outros produtos químicos e solventes utilizados eram de grau farmacêutico e foram utilizados tais como foram recebidos.

\subsection{Métodos}

\subsubsection{Preparação e caracterização das nanoemul- sões}

As nanoemulsões foram preparadas em triplicata, utilizando o método de emulsificação espontânea (Bouchemal et al 2004). Resumidamente, uma fase orgânica constituída por CTZ (50 mg), Span ${ }^{\circledR} 80$ (115 mg), óleo de melaleuca (500 $\mathrm{mg}$ ) e acetona $(25 \mathrm{~mL})$ foi adicionada a uma fase aquosa composta por Tween ${ }^{\circledR} 80$ (115 mg) e água ultra pura $(50 \mathrm{~mL})$, a qual permaneceu sob agitação moderada e constante, por 10 minutos. A formulação foi levada ao evaporador rotatório afim de eliminar o solvente orgânico e atingir um volume final de $50 \mathrm{~mL}$. Foram, também, preparadas formulações controle, sem 
a presença do fármaco. A concentração de CTZ e óleo de melaleuca foi de $1,0 \mathrm{mg} / \mathrm{mL}$ e $10 \mathrm{mg} / \mathrm{mL}$, respectivamente. As nanoemulsões foram acondicionadas em frascos de vidro âmbar e armazenadas à temperatura ambiente. As nanoemulsões foram denominadas: CTZNE (nanoemulsão contendo CTZ) e NE (nanoemulsão sem o fármaco).

Após a preparação e 30 dias de armazenamento, as nanoemulsões foram caracterizadas de acordo com os seguintes parâmetros: tamanho médio das gotículas, índice de polidispersão (PDI), potencial zeta, $\mathrm{pH}$, morfologia e teor de fármaco. Os testes foram realizados em triplicata, para cada lote de formulação.

O tamanho médio das gotículas e o índice de polidispersão foram determinados por espectroscopia de correlação de fótons (Zetasizer Nano ZS, MalvernInstruments, UK), a $25 \pm 2{ }^{\circ} \mathrm{C}$, após diluição de uma alíquota das amostras em água ultrapura (1:500). As medidas do potencial zeta foram realizadas no mesmo equipamento, por mobilidade eletroforética, após diluição das amostras em solução de $\mathrm{NaCl} 10 \mathrm{mM}$ (1:500).

A determinação do $\mathrm{pH}$ foi realizada diretamente nas formulações, utilizando-se potenciômetro previamente calibrado (SevenEasy, Mettler Toledo, São Paulo, Brasil) com soluções tampão pH 4,0 e 7,0.

A determinação do teor de CTZ foi realizada por cromatografia líquida de alta eficiência (CLAE), de acordo com metodologia descrita por Santos e colaboradores (2012), com modificações. Para a extração do fármaco, uma alíquota contendo $120 \mu \mathrm{L}$ das amostras foi diluída em $20 \mathrm{~mL}$ de metanol, submetida ao ultrassom por 10 minutos e, após, filtrada em membrana de celulose regenerada $(0,45 \mu \mathrm{m})$. Após este procedimento, as amostras foram analisadas em um cromatógrafo Shimadzu LC -10A, equipado com bomba modelo LC-10AD, detector SPD-10A e sistema controlador SCL-10A (Kyoto, Japão). A separação foi realizada utilizando uma coluna Phenomenex ${ }^{\circledR} \mathrm{C} 18(150 \times 4,60 \mathrm{~mm}, 5 \mu \mathrm{m})$ e pré-coluna C18 Phenomenex ${ }^{\circledR}$ Security Guard em temperatura ambiente. A fase móvel isocrática foi constituída por metanol e água (80:20, v/v), a uma vazão de $1,0 \mathrm{~mL} / \mathrm{min}$. O volume de injeção foi de $20 \mu \mathrm{L}$ e a detecção foi realizada a $229 \mathrm{~nm}$.

A taxa de associação do fármaco foi determinada pela técnica de ultrafiltração/centrifugação (Amicon ${ }^{\circledR}$ $10.000 \mathrm{MW}$, Millipore), a 2200 xG, durante 10 minutos, e o CTZ livre foi detectado no ultrafiltrado, por CLAE. A eficiência de encapsulamento (\%) foi calculada pela diferença entre a concentração total de fármaco e a concentração de fármaco no ultrafiltrado.

O método foi validado e os parâmetros analíticos utilizados foram especificidade, linearidade, precisão e exatidão (BRASIL, 2003; ICH, 2005). A especificidade do método foi avaliada por comparação dos cromatogramas obtidos para as amostras com (CTZ-NE) e sem o fármaco (NE), a fim de confirmar se os excipientes utilizados na preparação das formulações interferiram na quantificação do CTZ. A fim de avaliar a linearidade do método, cinco concentrações de CTZ $(2.0,4.0,6.0$, 8.0 e $10.0 \mu \mathrm{g} / \mathrm{mL}$ ), em triplicata, foram analisadas. A repetibilidade (precisão intradia) e precisão intermediária (entre dias) foram realizadas para seis amostras na mesma concentração $(6,0 \mu \mathrm{g} / \mathrm{mL})$, sob as mesmas condições experimentais, durante o mesmo dia e em dois dias diferentes, respectivamente. A exatidão foi determinada pelo teste de recuperação $(n=3)$, no qual o percentual de recuperação foi calculado pela diferença entre as áreas das concentrações obtidas com acréscimo ou não de solução padrão (AOAC, 2000).

\subsubsection{Avaliação da atividade antifúngica in vitro}

A avaliação da atividade antifúngica foi realizada pelo método microbiológico de difusão em ágar com pocinhos (Farmacopéia Brasileira V, 2010). O microrganismo empregado foi a levedura de C. albicans ATCC 10231, mantida em ágar Sabouraud-dextrose 4\% (SAD), por 48 horas, a temperatura de $25 \pm 2{ }^{\circ} \mathrm{C}$ (tubo inclinado). O inóculo utilizado foi a $1 \%(\mathrm{v} / \mathrm{v})$, após a preparação da suspensão do microrganismo em solução salina estéril, a uma concentração de $25 \pm 2 \%$ de transmitância, lida em espectrofotômetro (Pró-Análise, modelo UV-1800 PC, Porto Alegre, Brasil), a $580 \mathrm{~nm}$ (contendo, aproximadamente, $7,0 \times 10^{4} \mathrm{UFC} / \mathrm{mL}$ ). Uma alíquota das nanoemulsões (CTZ-NE e NE) foi diluída em água purificada para se obter uma concentração final de 4,0 $\mu \mathrm{g} / \mathrm{mL}$ de CTZ. Para comparação, uma solução do fármaco foi empregada, na mesma concentração (CTZ-S). Para cada amostra utilizaram-se grupos de seis placas de Petri, as quais continham $25 \mathrm{~mL}$ de meio SAD como camada base e $5 \mathrm{~mL}$ de inóculo. Alíquotas de $100 \mu \mathrm{L}$ foram colocadas, individualmente, em cada orifício $(9,5$ $\mathrm{mm}$ de diâmetro). As placas foram incubadas em estufa, à temperatura de $25 \pm 5^{\circ} \mathrm{C}$, por 48 horas, e após este período, o diâmetro dos halos de inibição foi determinado $(\mathrm{mm})$ com o auxílio de paquímetro digital (Paquímetro Eletrônico Digital Starrett, 727).

\subsubsection{Preparação dos hidrogéis}

Hidrogéis de Carbopol ${ }^{\circledR}$ Ultrez foram preparados, em triplicata, após a dispersão do polímero diretamente nas nanoemulsões (HG-CTZ-NE), com auxílio de gral e pistilo, a fim de obter a concentração de fármaco de $1,0 \mathrm{mg} / \mathrm{g}$. As formulações foram neutralizadas com trietanolamina e imidazolidinil ureia foi utilizado como conservante. Para comparação, hidrogéis contendo as nanoemulsões sem o fármaco (HG-NE) e contendo o fármaco não associado (HG-CTZ) foram, também, preparados e avaliados. A composição quali-quantitativa dos hidrogéis está descrita na Tabela 1. 
Tabela 1 - Composição quali-quantitativa dos hidrogéis

\begin{tabular}{lccc}
\hline Componentes & HG-CTZ-NE & HG-NE & HG-CTZ \\
\hline Carbopol $^{\circledR}$ Ultrez & $200 \mathrm{mg}$ & $200 \mathrm{mg}$ & $200 \mathrm{mg}$ \\
Trietanolamina & $50 \mathrm{mg}$ & $50 \mathrm{mg}$ & $50 \mathrm{mg}$ \\
NE-CTZ & $50 \mathrm{~mL}$ & - & - \\
NE & - & $50 \mathrm{~mL}$ & - \\
Clotrimazol & - & - & $50 \mathrm{mg}$ \\
Imidazolidinil & $150 \mathrm{mg}$ & $150 \mathrm{mg}$ & $150 \mathrm{mg}$ \\
ureia & - & - & $12,5 \mathrm{~mL}$ \\
Etanol & - & - & $37,5 \mathrm{~mL}$ \\
Água ultra pura & & &
\end{tabular}

\subsubsection{Caracterização físico-química dos hidrogéis}

As formulações foram avaliadas em relação as suas características físico-químicas e os testes realizados compreenderam: análise macroscópica (cor, odor e aspecto), teor de CTZ, tamanho das gotículas, espalhabilidade, vicosidade e propriedades reológicas.

A determinação do teor de CTZ foi realizada por CLAE. Para a extração do fármaco, uma alíquota de $0,12 \mathrm{~g}$ das amostras foi pesada e diluída em $20 \mathrm{~mL}$ de metanol (a fim de obter uma concentração de 6,0 $\mu \mathrm{g} / \mathrm{mL}$ ), submetida a agitação magnética por 30 minutos, centrifugada por 5 minutos e, após, filtrada em membrana de celulose regenerada $(0,45 \mu \mathrm{m})$. $\mathrm{O}$ método foi validado e os parâmetros analíticos utilizados foram linearidade, especificidade e precisão (BRASIL, 2003; ICH, 2005).

A medida do $\mathrm{pH}$ dos hidrogéis foi realizada em potenciômetro, após dispersão dos hidrogéis em água $(10 \%, \mathrm{~m} / \mathrm{v})$. As análises foram realizadas em triplicata.

O tamanho médio das gotículas e o índice de polidispersão (PDI) foram determinados por espectroscopia de correlação de fótons (Zetasizer Nano ZS, MalvernInstruments, UK), a $25 \pm 2{ }^{\circ} \mathrm{C}$, após diluição de uma alíquota dos hidrogéis em água (1:500).

As características reológicas e a viscosidade das formulações em estudo foram determinadas com o auxílio de viscosímetro rotacional Brookfield LVDV-II+ Pro, empregando-se como cilindro de medição SC4-25. As análises foram realizadas a temperatura de $25 \pm 1 \stackrel{\circ}{ } \mathrm{C}$. Os reogramas foram analisados usando diferentes modelos de fluxo: Bingham $\left(\tau=\tau_{0}+\eta \gamma\right)$, Casson $\left(\tau=\tau_{0}^{0,5}+\eta^{0,5} \gamma^{0,5}\right)$, Ostwald $\left(\tau=\kappa \gamma^{\mathrm{n}}\right)$ e Herschel-Bulkley $\left(\tau=\tau_{0}+\kappa \gamma^{\mathrm{n}}\right)$, onde: $\tau=$ tensão de cisalhamento; $\tau_{0}=$ tensão de cisalhamento limite; $\eta=$ viscosidade; $\mathrm{n}=$ índice de fluxo; $\kappa=$ índice de consistência e $\gamma=$ taxa de cisalhamento (Kim et al., 2003).

A espalhabilidade dos hidrogéis foi realizada de acordo com o método das placas paralelas (Borguetti e Knorst, 2006). Os resultados foram expressos em relação à espalhabilidade da amostra e os pesos adicionados, de acordo com a equação 1 , sendo que os mesmos correspondem à média de três determinações. $\mathrm{O}$ fator de espalhabilidade foi, também, calculado (equação 2):

(1) $\mathrm{Ei}=\mathrm{d}^{2} \times \pi / 4$

onde: $\mathrm{Ei}$ = espalhabilidade da amostra para um determinado peso i $\left(\mathrm{mm}^{2}\right) ; \mathrm{d}=$ diâmetro médio $(\mathrm{mm})$.

(2) $\mathrm{Fe}=\mathrm{Ei} / \mathrm{P}$

onde: $\mathrm{Fe}=$ fator de espalhabibidade da amostra $\left(\mathrm{mm}^{2} / \mathrm{g}\right)$; $\mathrm{Ei}=$ área de espalhabilidade máxima $\left(\mathrm{mm}^{2}\right) ; \mathrm{P}=$ peso total adicionado $(\mathrm{g})$.

\subsubsection{Avaliação da liberação in vitro do fármaco a partir dos hidrogéis}

Um estudo de liberação in vitro foi realizado utilizando células de difusão vertical do tipo Franz ( $\mathrm{n}=$ 6) com área de difusão de $1,77 \mathrm{~cm}^{2}$ e $7,0 \mathrm{~mL}$ de meio receptor composto por água:etanol (70:30, v/v), a $37 \pm$ $0,5^{\circ} \mathrm{C}$, empregando uma membrana sintética de acetato de celulose (porosidade de $0,45 \mu \mathrm{m}$ ) como barreira para liberação. Uma amostra de hidrogel contendo $500 \mathrm{mg}$ de CTZ livre ou associado à nanoemulsãofoi espalhada na superfície da membrana e, após intervalos de tempos de $1,2,4,6$ e 8 horas, alíquotas do meio receptor $(200 \mu \mathrm{L})$ foram coletadas, repondo-se a mesma quantidade de meio fresco e o experimento foi realizado em condições sink. A quantidade de CTZ liberada foi determinada por CLAE, utilizando as condições cromatográficas descritas no item 2.2; entretanto, devido à necessidade do uso de concentrações menores, o método foi validado e os parâmetros de linearidade e precisão foram avaliados (BRASIL, 2003; ICH, 2005). Os perfis de liberação in vitro 
do fármaco, a partir dos hidrogéis, foram analisados mediante modelagem matemática (MicroMathScientist ${ }^{\circledR}$ for WindowsTM), empregando o modelo de Higuchi (Siepmann e Peppas, 2001).

\subsubsection{Análise estatística}

As análises foram realizadas em triplicata e os resultados foram expressos como média e desvio padrão. Para avaliação dos resultados, a análise de variância (ANOVA) foi utilizada, seguida do teste de Tukey, a um nível de significância de 5\%.

\section{RESULTADOS}

\subsection{Caracterização físico-química e estabilida- de das nanoemulsões}

As nanoemulsões, após a preparação, apresentaramse macroscopicamente homogêneas, com aspecto levemente branco leitoso e reflexo azulado (efeito Tyndall), bem como odor característico do óleo de melaleuca. Os resultados obtidos na caracterização das nanoestruturas encontram-se na tabela 2.

Tabela 2 - Caracterização físico-química das nanoemulsões contendo CTZ $(1,0 \mathrm{mg} / \mathrm{mL})$ e óleo de melaleuca (CTZ-NE) e sem o fármaco (NE) $(n=3$, média $\pm \mathrm{s}$ )

\begin{tabular}{lcc}
\hline Parâmetros & CTZ-NE & NE \\
\hline $\mathrm{pH}$ & $4,83 \pm 0,01$ & $4,40 \pm 0,05$ \\
$\begin{array}{l}\text { Tamanho } \\
\text { médio }(\mathrm{nm})\end{array}$ & $370 \pm 42$ & $160 \pm 17$ \\
PDI & $0,13 \pm 0,05$ & $0,13 \pm 0,01$ \\
$\begin{array}{l}\text { Potencial zeta } \\
(\mathrm{mV})\end{array}$ & $-7,1 \pm 1,3$ & $-8,3 \pm 1,3$ \\
Teor $(\mathrm{mg} / \mathrm{mL})$ & $1,017 \pm 0,01$ & - \\
\hline
\end{tabular}

Quanto ao teor de fármaco, as amostras apresentaram valor próximo ao teórico $(1,02 \pm 0,92 \mathrm{mg} / \mathrm{mL})$ e a eficiência de encapsulamento foi de, aproximadamente, $100 \%$.

$\mathrm{O}$ método analítico mostrou-se linear na faixa de concentração de 2 a 10 mg/mL (y = 74706x-966,22), com regressão linear significativa ( $\mathrm{F}$ calculado $=11745,54<\mathrm{F}$ crítico $=4,96, \mathrm{p}<0,05)$ e sem desvio de linearidade $(\mathrm{F}$ calculado $=3,60<\mathrm{F}$ crítico $=3,71, \mathrm{p}>0,05)$; específico, uma vez que não houve interferência dos excipientes e do óleo de melaleuca no pico cromatográfico do CTZ; preciso (DPR $=1,70 \%$ e $0,56 \%$ para repetibilidade e precisão intermediária, respectivamente) e exato (percentual de recuperação de 98 a 101\%).

Após 30 dias de armazenamento à temperatura ambiente e ao abrigo da luz, as formulações mantiveram suas características macroscópicas iniciais, sem precipitação e alterações de cor ou aspecto. A figura 1 demonstra um comparativo entre os valores obtidos para o teor, o tamanho médio das gotículas, PDI e potencial zeta, após a preparação e 30 dias de armazenamento.

Quanto ao $\mathrm{pH}$, uma redução em relação aos valores iniciais $(p<0,05)$ foi observada, tanto para as nanoemulsões com CTZ, como para as que não continham o fármaco (de 4,83 $\pm 0,01$ a 4,68 $\pm 0,07$ para a CTZ-NE, e de $4,40 \pm 0,05$ a $3,83 \pm 0,06$ para a NE).

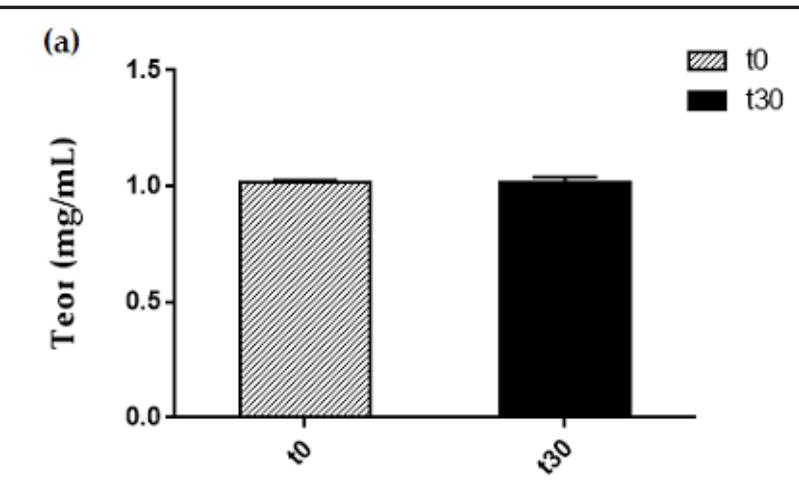

(b)
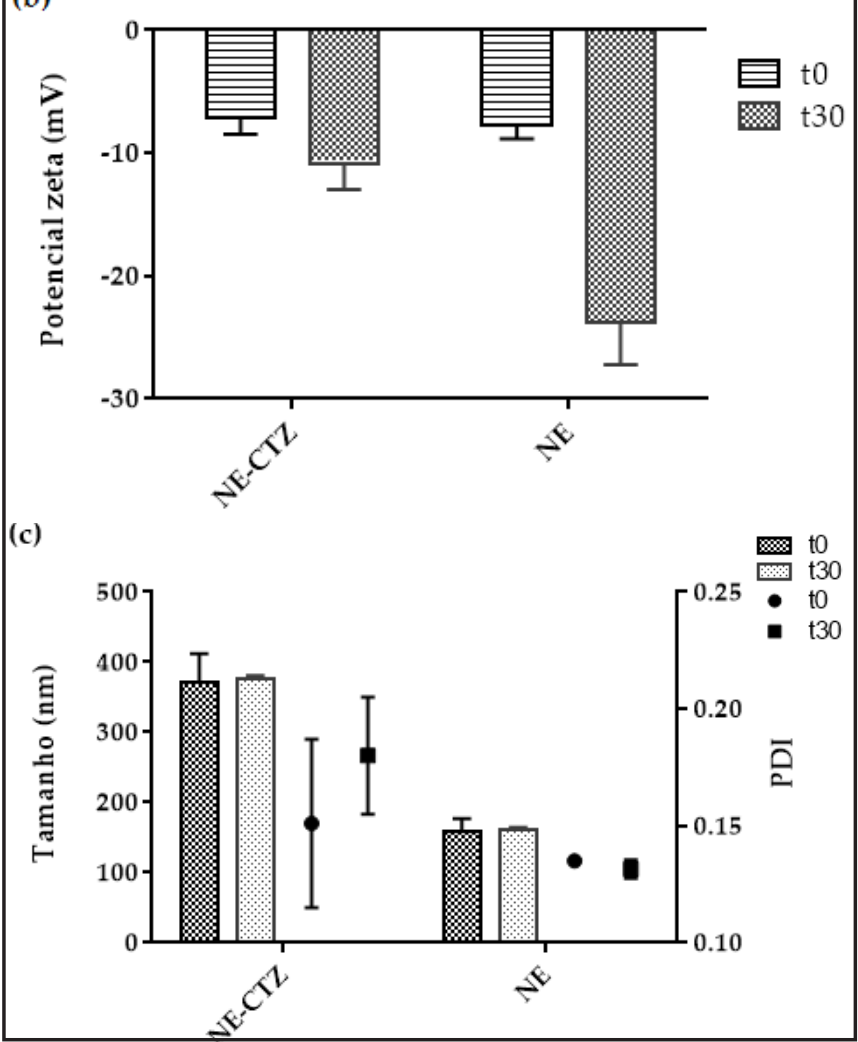

Figura 1 - Avaliação do teor de fármaco (a), potencial zeta (b), tamanho médio das gotículas e índice de polidispersão (PDI) (c) das nanoemulsões contendo CTZ e óleo de melaleuca (CTZ-NE) e das nanoemulsões sem o fármaco (NE) determinados no estudo de estabilidade $\left(t_{0}=\right.$ após preparação; $\mathrm{t}_{30}=$ após 30 dias de armazenamento). 


\subsection{Atividade antifúngica in vitro}

Neste trabalho, a atividade antifúngica do fármaco em nanoemulsão (CTZ-NE) e em solução (CTZ-S) foi avaliada frente à levedura de C. albicans. A partir dos resultados foi possível observar que não houve diferença significativa $(p>0,05)$ entre os halos de inibição verificados para o fármaco não associado (CTZ-S: 21,8 \pm 1,1 $\mathrm{mm}$ ) em comparação com o fármaco nanoestruturado (CTZ-NE: 20,78 \pm 1,12 mm). Em relação à nanoemulsão sem o fármaco (NE), preparada somente com o óleo de melaleuca, não foram observados halos de inibição.

\subsection{Caracterização físico-química dos hidro- géis}

Quanto à análise macroscópica, os hidrogéis contendo as nanoemulsões (HG-CTZ-NE) e a formulação sem o fármaco (HG-NE) apresentaram características organolépticas satisfatórias, com aspecto homogêneo e brilhante, odor característico do óleo de melaleuca e coloração branca semelhante a um gel-creme, para o HG-NE-CTZ, e coloração levemente esbranquiçada para HG-NE. As formulações contendo o fármaco não associado (HG-CTZ) apresentaram aspecto homogêneo, brilhante e transparente. Os resultados obtidos na caracterização físico-química das formulações estão apresentados na tabela 3 .

Na figura 2 estão representados os reogramas dos hidrogéis. Em relação ao tipo de escoamento, o modelo que melhor descreveu o fluxo dos hidrogéis foi o de Casson, apresentando uma correlação maior que 0,99.

Após estabelecido o modelo de fluxo, os valores de viscosidade $(\eta)$ e de tensão de cisalhamento limite $\left(\tau_{0}\right)$ foram calculados (tabela 4 ) e diferenças não foram verificadas entre as formulações $(p>0,05)$.

No que se refere à espalhabilidade, após avaliar a área de espalhabilidade em função do peso adicionado, verificou-se que os hidrogéis apresentaram valores de espalhabilidade semelhantes ( $>00,05)$ (tabela 4$)$.

Tabela 3 - Caracterização físico-química dos hidrogéis ( $\mathrm{n}=3$, média $\pm \mathrm{s}$ )

\begin{tabular}{lcccc}
\hline Formulação & $\begin{array}{c}\text { Tamanho } \\
\text { médio }(\mathbf{n m})\end{array}$ & PDI & pH & Teor $(\mathbf{m g} / \mathbf{g})$ \\
\hline HG-CTZ-NE & $424 \pm 40^{\mathrm{a}}$ & $0,16 \pm 0,12^{\mathrm{a}}$ & $6,27 \pm 0,46^{\mathrm{a}}$ & $1,00 \pm 0,01^{\mathrm{a}}$ \\
HG-NE & $243 \pm 5^{\mathrm{b}}$ & $0,24 \pm 0,01^{\mathrm{a}}$ & $6,46 \pm 0,17^{\mathrm{a}}$ & - \\
HG-CTZ & - & - & $6,36 \pm 0,19^{\mathrm{a}}$ & $1,02 \pm 0,01^{\mathrm{a}}$ \\
\hline
\end{tabular}

Letras iguais na mesma coluna indicam que não houve diferença estatística ( $p>0,05)$.

Tabela 4 - Valores de viscosidade $(\eta)$, tensão de cisalhamento limite $\left(\tau_{0}\right)$ e fator de espalhabilidade (FE) para os hidrogéis contendo as nanoemulsões com CTZ (HG-CTZ-NE) e sem o fármaco (HG-NE), bem como para o hidrogel com o CTZ não associado (HG-CTZ)

\begin{tabular}{llll}
\hline Formulação & $\eta(\mathbf{m P a} . \mathbf{s})$ & $\boldsymbol{\tau} 0\left(\mathbf{D} / \mathbf{c m}^{2}\right)$ & FE $\left(\mathbf{m m}^{2} \mathbf{g}\right)$ \\
\hline HG-CTZ-NE & $24167 \pm 6393$ & $171 \pm 1,56$ & $4,24 \pm 0,17$ \\
HG-NE & $16050 \pm 1533$ & $302 \pm 35$ & $4,63 \pm 0,21$ \\
HG-CTZ & $14015 \pm 4180$ & $369 \pm 59$ & $4,70 \pm 0,51$ \\
\hline
\end{tabular}




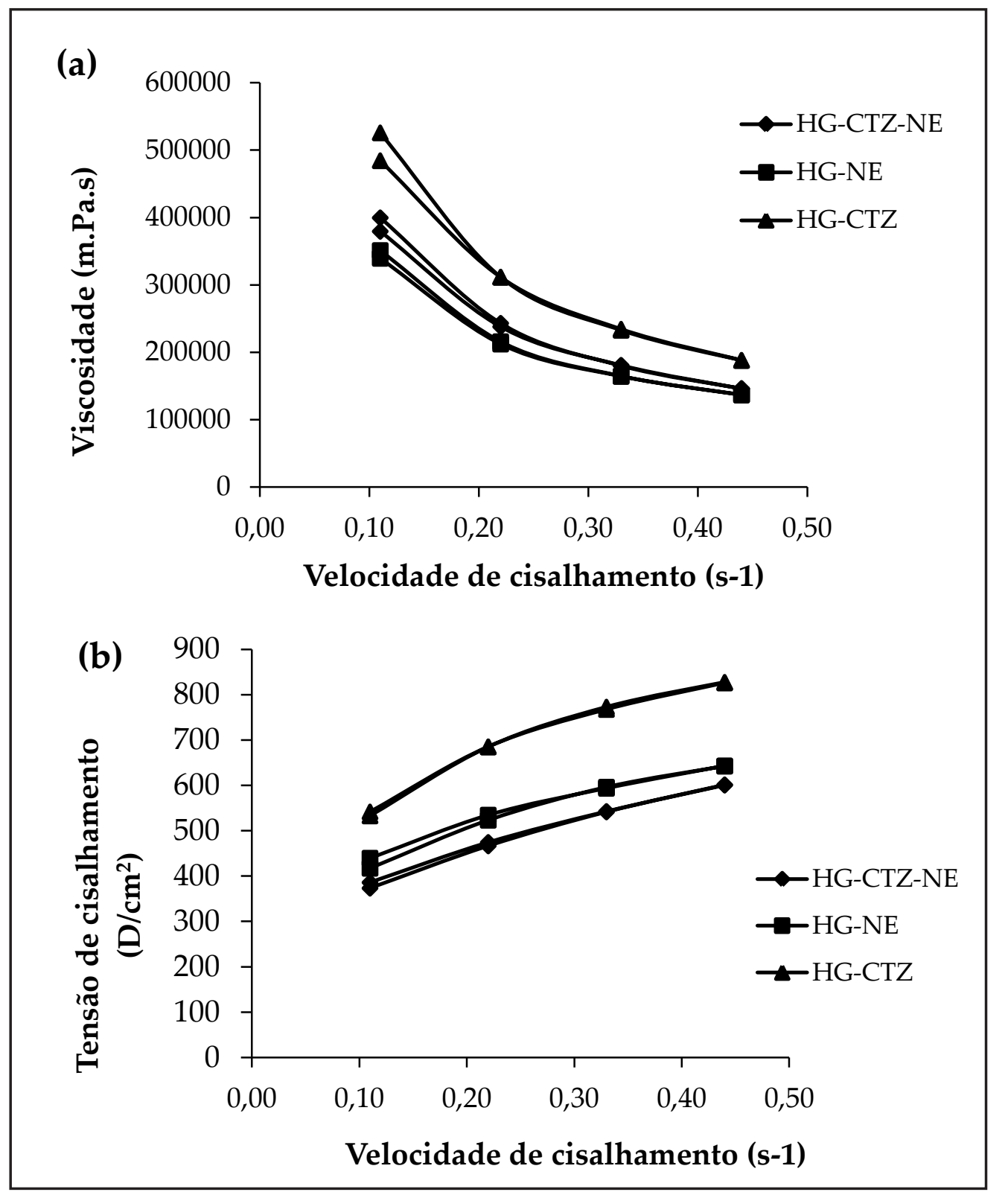

Figura 2 - Representação gráfica da viscosidade em relação à taxa de cisalhamento (a) e da tensão de cisalhamento versus velocidade de cisalhamento dos hidrogéis (b).

3.4. Avaliação da liberação in vitro do fármaco a partir dos hidrogéis

O método analítico para a quantificação do CTZ foi validado quanto à linearidade e precisão, mostrandose linear na faixa de concentração de 0,1 a $20 \mu \mathrm{g} / \mathrm{mL}$ e preciso ( $\mathrm{DPR}=1,51 \%)$. A figura 3 representa o perfil de liberação do fármaco a partir de hidrogel contendo a nanoemulsão (HG-CTZ-NE) e do hidrogel contendo o fármaco não associado (HG-CTZ). Em oito horas, 22,95 $\pm 5,49 \mu \mathrm{g} / \mathrm{cm}^{2}(4.59 \%)$ de CTZ foi liberado a partir do HG-CTZ, enquanto que uma quantidade de 11,80 \pm $1,89 \mu \mathrm{g} / \mathrm{cm}^{2}(2.36 \%)$ foi liberada a partir do HG-CTZ$\mathrm{NE}$, demonstrando a capacidade da nanoemulsão em controlar a liberação in vitro do fármaco.A partir da modelagem matemática, a constante de velocidade de liberação para os hidrogéis foi calculada e os valores encontrados corresponderam a 4,17 $\pm 0,67 \mathrm{~h}^{-1}$ e 8,11 \pm 1,94 $\mathrm{h}^{-1}$ para HG-CTZ-NE e HG-CTZ, respectivamente. 


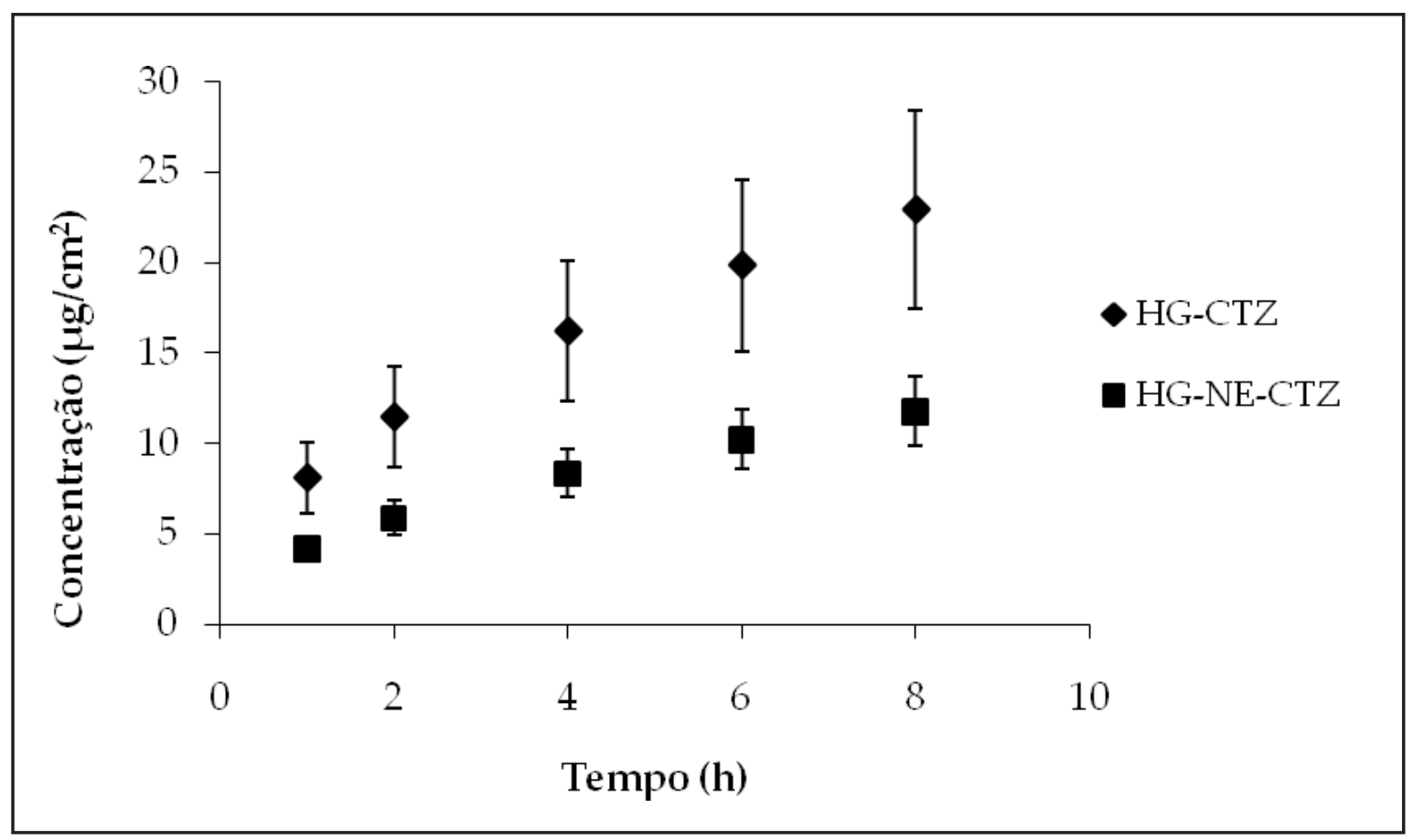

Figura 3. Perfil de liberação in vitro dos hidrogéis contendo CTZ associado à nanemulsão (HG-CTZ-NE) e não associado (HG-CTZ).

\section{DISCUSSÃO}

Em relação à caracterização físico-química, as nanoemulsões apresentaram-se como um líquido branco leitoso e reflexo azulado, em função do movimento Browniano das moléculas (SCHAFFAZICK et al., 2003, MORA-HUERTAS et al., 2010). As formulações apresentaram tamanho das gotículas na faixa manométrica e baixo valor de polidispersão, indicando a homogeneidade do sistema. Diferenças significativas foram observadas em relação às nanoemulsões sem o fármaco $(\mathrm{p}<0,05)$, demonstrando que a presença do mesmo pode ter influenciado o tamanho médio das gotículas. O pH das amostras encontrou-se na faixa ácida devido as características dos componentes das formulações. A adição do fármaco promoveu um pequeno aumento no $\mathrm{pH}$ das nanoemulsões $(p<0,05)$, devido, provavelmente, ao caráter básico do CTZ. O teor de fármaco foi próximo ao valor teórico, indicando que o processo de preparação não acarretou na degradação ou perda da substância ativa.

Após 30 dias de armazenamento a temperatura ambiente, diferenças significativas não foram observadas para as nanoemulsões contendo CTZ em relação ao tamanho, potencial zeta, polidispersão e teor de CTZ. No que se refere ao $\mathrm{pH}$, uma pequena redução em relação ao valor inicial foi verificada $(<1,0)$. Este efeito corrobora com os resultados encontrados por Flores e colaboradores (2011), os quais verificaram uma diminuição do pH de nanoemulsões contendo óleo de melaleuca após 60 dias de armazenamento e atribuem este efeito ao maior contato do óleo com a fase aquosa das formulações, conduzindo a degradação de alguns dos seus componentes. Em outro estudo, Hashem e colaboradores (2011) atribuíram a redução do $\mathrm{pH}$ de microemulsões de CTZ na forma líquida e de gel à degradação ácida do fármaco.

A fim de verificar se o método de preparação e o nanoencapsulamento poderia alterar a atividade microbiológica do fármaco, o estudo da atividade antifúngica frente a levedura de $C$. albicans foi realizado. Tendo em vista que os halos de inibição do fármaco em solução e do fármaco associado à nanoestrutura foram semelhantes estatisticamente, foi possível observar que a inclusão do CTZ em nanoemulsão não alterou a sua atividade. Ainda, o fato de que a nanoemulsão contendo somente o óleo de melaleuca não apresentar halo de inibiçãopode estar relacionado com à elevada lipofilicidade do óleo, dificultando a sua difusão no meio de cultura, bem como a concentração utilizada.

A atividade de fármacos antifúngicos vinculados a nanoestruturas tem sido estudada. Padmavathy e Vijayaraghavan (2008) avaliaram a atividade antibacteriana de micro e nanopartículas de $\mathrm{ZnO}$, as quais demonstraram maior ação devido a maior área superficial em contato com o microrganismo. Santos e colaboradores (2013) avaliaram a atividade de nanocápsulas de CTZ frente a cepas de $C$. albicans suscetíveis ao fluconazol e cepas de C. glabrata suscetíveis e resistentes ao fluconazol. Neste estudo, as nanocápsulas mantiveram a atividade do fármaco frente às espécies de Candidaquando comparadas com soluções metanólicas contendo o fármaco em sua forma livre e soluções metanólicas contendo triglicerídeos de cadeia média. 
A fim de obter uma forma farmacêutica adequada para aplicação tópica, neste trabalho uma preparação semissólida do tipo hidrogel foi preparada e avaliada em relação as suas propriedades físico-químicas. Os hidrogéis são veículos aquosos que apresentam sensorial agradável e de fácil aplicação na pele (BUWALD A et al., 2014). Ainda, a inclusão de substâncias lipofílicas na fase interna de nanoemulsões, possibilita que estas possam ser incorporadas em bases semissólidas do tipo gel. Os hidrogéis apresentaram características adequadas, com pH compatível para aplicação tópica (KIM et al., 2006).

A avaliação das características reológicas de formulações semissólidas pode auxiliar no prognóstico dos efeitos da formulação e do processamento nas características do produto, bem como, na avaliação de sua qualidade e estabilidade (KIM et al., 2003).Com base nos reogramas das formulações, foi possível observar um comportamento de fluxo Não-newtoniano, no qual não há uma relação linear entre a taxa de cisalhamento e a tensão de cisalhamento (figura $2 b$ ). Nos sistemas não -newtonianos, os fluidos podem apresentar características plásticas, pseudoplásticas e dilatantes (KIM et al., 2003). A partir da modelagem matemática, o modelo de Casson foi verificado para as amostras em estudo, e descreve o comportamento plástico dos sistemas, caracterizado por apresentar uma tensão de cisalhamento inicial para iniciar o fluxo das camadas moleculares. Este resultado vai de encontro aos obtidos por Flores e colaboradores (2015) para hidrogéis de Carbopol $^{\circledR}$ Ultrez contendo nanoemulsão e nanocápsula de óleo de melaleuca.

No que diz respeito à espalhabilidade, não foram observadas diferenças significativas entre os fatores de espalhabilidade dos hidrogéis $(p>0,05)$ (tabela 4). A determinação da espalhabilidade também é importante no desenvolvimento de formulações semissólidas, pois verifica a capacidade da formulação em espalhar sobre uma superfície, relacionada com a sua aplicação no local desejado (Garg et al., 2002).

No estudo de liberação in vitro utilizando membrana sintética, verificou-se que a associação do CTZ à nanoemulsão (HG-CTZ) possibilitou uma liberação mais lenta do mesmo em comparação ao fármaco não associado (HG-CTZ-NE). A partir dos resultados, a constante de velocidade de liberação do HG-CTZ foi duas vezes maior que a calculada para o HG-CTZ-NE $(p<0,05)$. Este resultado corrobora com outros trabalhos existentes na literatura, que demonstram a capacidade dos nanocarreadores em controlar a liberação de substâncias. Em estudo realizado por Marchiori e colaboradores (2010), obteve-se uma liberação controlada da dexametasona em intervalos de 2 e 24 horas a partir de hidrogéis de Carbopol ${ }^{\circledR}$ Ultrez contendo o fármaco associado à nanocápsulas com relação aos hidrogéis com o fármaco livre. No ano seguinte, Fontana e colaboradores relataram que hidrogéis de Carbopol ${ }^{\circledR}$ Ultrez 10 NF contendo propionato de clobetasol em nanoemulsões e nanocápsulas apresentaram uma liberação mais lenta do que o hidrogel contendo o fármaco não associado.

Assim, a liberação controlada do CTZ por parte da nanoemulsão, apresenta-se como uma vantagem com relação ao tratamento tópico, visto que uma liberação sustentada do fármaco permite a manutenção da dose no local aplicado ao longo do tempo.

\section{CONCLUSÃO}

Este trabalho demonstrou a viabilidade em preparar hidrogéis de Carbopol ${ }^{\circledR}$ Ultrez contendo CTZ e óleo de melaleuca associados à nanoemulsão. As nanoemulsões apresentaram características físico-químicas satisfatórias e se mantiveram estáveis após 30 dias de armazenamento a temperatura ambiente. Além disso, a manutenção da atividade antifúngica do fármaco após a sua inclusão em nanoemulsão foi verificada frente à levedura de $C$. albicans. Os hidrogéis contendo as nanoemulsões apresentaram pH compatível com aplicação tópica, teor de fármaco próximo ao teórico e características reológicas plásticas. Ainda, a nanoemulsão foi capaz de promover uma liberação controlada do fármaco, o que permite reduzir o número de aplicações do mesmo no local de ação, proporcionando maior comodidade ao paciente.

\section{AGRADECIMENTOS}

Os autores agradecem à FAPERGS pelo apoio financeiro.

\section{REFERÊNCIAS}

Anton N, Benoit JP, Saulnier P. Design and production of nanoparticles formulated from nano-emulsion templates - A review. J Control Release. 2008;128:185-199.

AOAC. Association of Official Analitycal Chemists. Official Methods of Analysis. Gaithersburg, MD. 2000; Ed 17, v. 1.

Borguetti GS, Knorst MT. Desenvolvimento e avaliação da estabilidade física de loções O/A contendo filtros solares. Rev Bras Cienc Farm. 2006;42(4):531-537.

Bouchemal K, BriançonS, Pierrer E, Fessi H. Nano-emulsion formulation using spontaneous emulsification: solvent, oil and surfactant optimisation. Int J Pharm. 2004;280:241-251.

BRASIL. Resolução - RE no 899 de 29 de maio de 2003. Guia para validação de métodos analíticos e bioanalíticos. Diário Oficial da União, Brasília, DF, 02 de junho de 2003.

British Pharmacopeia, British Pharmacopeia Comission, Londres: Her Majesty Stationary Office. 2010; v.1. 
BurgessMA, Bodey GP. Clotrimazole (Bay b 5097): I Vitro and Clinical Pharmacological Studies. Antimicrob Agents Chemother. 1972;2(6):423-426.

Buwalda SJ, Boere KWM, Dijkstra PJ, Feijen J,Vermonden T, Hennink WE. Hydrogels in a historical perspective: From simple networks tosmart materials. J Control Release. 2014;190:254-273.

Carson CF,Hmmer KA, Riley TV. Melaleucaalternifolia(Tea Tree) Oil: a Review of Antimicrobial and Other Medicinal Properties. Clin Microbiol Rev. 2006;19(1):50-62.

Chang JY, Oh Y-K, Kong HS, Kim EJ, Jang DD, Nam $\mathrm{KT}$ et al. Prolonged antifungal effects of clotrimazolecontaining mucoadhesivethermosensitive gels on vaginitis. J Control Release.2002;82:39-50.

Farmacopeia Brasileira. V.1, 5. ed. Brasília: Anvisa, 2010.

Flores FC, Ribeiro RF, Ourique AF, Pohlmann AR, Beck RCR, Guterres SS et al. Nanostructurated systems containing an essential oil: protection against volatilization. Quim Nova. 2011;34(6):968-972.

Flores FC, LimaJA, Silva CR, Benvegnú D, Ferreira J, Burger ME et al. Hydrogels containing nanocapsules and nanoemulsions of tea tree oil provide antiedematogenic effect and improved skin wound healing. J Nanosci Nanotechnol. 2015;15(1):800-809.

Fontana MC, Rezer JFP, Coradini K, Leal DBR, Beck RCR. Improved efficacy in the treatment of contact dermatitis in rats by a dermatological nanomedicine containing clobetasol propionate. Eur J Pharm Biopharm. 2011;79:241-249.

Garg A,AggarwalD, Garg S, Singla A. Spreading of semisolid formulations: an update. Pharm Technol. 2002;26:84-105.

Georgopapadakou NH. Antifungals: mechanism of action and resistance, established and novel drugs. Curr Opin Microbiol. 1998;1:547-557.

Gupta AK, Einarson TR, Summerbell RC, Shear NH. An overview of topical antifungal therapy in dermatomycoses. A North American perspective. Drugs. 1998;55(5):645-74.

Guterres SS, Alves MP, Pohlmann AR. Polymeric nanoparticl

Santos SS, Ferreira LM, Schaffazick SR, Cruz L. A simple RP-HPLC method for the determination of clotrimazole from acrylic nanocapsule suspensions. Lat Am J Pharm. 2012;31(3):482-486.

Santos SS, Lorenzoni A, Ferreira LM, Mattiazzi J, Adams AIH, Denardi LB et al. Clotrimazole-loaded Eudragit ${ }^{\circledR}$ RS100 nanocapsules: Preparation, characterization and in vitro evaluation of antifungal activity against Candida species. Mat Sci Eng C. 2013;33:1389-1394.

Schaffazick RS, Guterres SS, Freitas LL, Pohlmann AR. Caracterização e estabilidade físico-química de sistemas poliméricos nanoparticulados para administração e fármacos. Quim Nova. 2003;26(5):726-737.

Siepmann J, Peppas NA. Modeling of drug release from delivery systems based on hydroxypropyl methylcellulose (HPMC). Adv Drug Deliv Rev. 2001;48:139-157.

Sobel JD. Vulvovaginal candidosis. L a nce t. $\quad 2007 ; 369: 1961-71$.

Sonneville-Aubrun O, Simonnet JT, L'alloret F. Nanoemulsions: a new vehicle for skincare products. Adv Colloid Interf Sci. 2004;108 -109:145-149.

Souto EB, Müller RH. Rheological and in vitro release behaviour of clotrimazole-containing aqueous SLN dispersions and commercial creams. Pharmazie 2007;62(7):505-509. 